\title{
Discrete Riccati equation for systems with singular transition matrix
}

\author{
Toru Fujinaka and Hiroshi Shibata \\ Department of Computer and Systems Sciences \\ Osaka Prefecture University \\ 1-1, Gakuen-cho, Sakai, Osaka, 599-8531 Japan \\ fuji@cs.osakafu-u.ac.jp
}

\begin{abstract}
We consider the discrete algebraic Riccati equation with singular coefficient matrix in relation to the linear quadratic regulator problem. It is shown that the positive definite solution of the Riccati equation can be obtained via a similar equation of reduced size. We derive the result by manipulating the performance index of the associated optimal regulator problem. This is an extension to a similar result in which some restriction applies to the form of the weighting matrix in the performance index.
\end{abstract}

\section{Introduction}

Various properties of the Riccati equation have been extensively studied, e.g., [1] and [2], and numerical algorithms have also been developed to solve the Riccati equation[3] with sufficient accuracy.

We focus our attention on the discrete-time Riccati algebraic equation (DARE) arising from the discretetime linear quadratic regulator (DLQR) problem where the coefficient matrix representing the state transition of the system is singular. This typically happens when the controlled system has time delay in its input or output. There is no difficulty in formulating the DLQR problem, and numerical algorithm for solving the DARE with singular transition matrix is available[4]. However, singularity of the transition matrix leads to larger dimension of the coefficient matrices, which may cause computational difficulties.

When the singularity of the transition matrix is due to the time delay in control, it has been shown [5] that the DARE associated with the DLQR problem can be solved via a DARE of smaller size. The result in [6], [7] can be applied to systems with time delay in the input, output, and/or anywhere along the signal path.
The size of the DARE to be actually solved becomes that of the system without time delay. These results depend on the special structure of the time-delay system. Moreover, there is a restriction in the form of the weighting matrix in the performance index to be minimized.

However, singularity of the state transition matrix in the DARE does not always arise from time delays. The DLQR problem with a cross-product term between the state and input of the performance index is converted to an equivalent DLQR problem without the cross term, by which the transition matrix may become singular. In the $\mathcal{H}_{2}$-optimal control problem the transition matrix of the generalized plant may or may not be singular regardless of the singularity of the transition matrix of the controlled plant. In these cases no special structure in the coefficient matrices may be expected, and conventional results developed for systems with time delay cannot be applied.

The present paper covers more general case of singular transition matrix in the DARE, and we show that the positive definite solution of the DARE can be obtained via a DARE of smaller size in general. The only assumption on the state transition matrix is that it is singular. Accordingly, no restriction on the structure of the positive definite weighting matrix is posed. The result we derive is different from the structure algorithm presented in [8], where the rank deficiency of the positive semidefinite solution is relevant. We will derive a formula similar to that shown in [9], where the Riccati difference equation associated with finite-time DLQR problem is considered.

The rest of the paper is organized as follows. In Section 2 we will formulate the problem of size reduction and introduce the results already known for time-delay systems. Section 3 presents the development and main result, and a numerical example is shown in Section 4, followed by the conclusions in Section 5 . 


\section{Problem Formulation}

\section{Consider the DARE}

$$
X=A^{T} X A+Q-A^{T} X B\left(R+B^{T} X B\right)^{-1} B^{T} X A
$$

which arises from the DLQR problem of minimizing the performance index

$$
J=\sum_{k=0}^{\infty}\left\{x^{T}(k) Q x(k)+u^{T}(k) R u(k)\right\}
$$

subject to the system dynamics given by

$$
x(k+1)=A x(k)+B u(k), \quad x(0)=x_{0}
$$

where the state transition matrix $A$ is singular for some reason.

Under a standard assumption that the pair $(A, B)$ is stabilizable and the weighting matrices $Q$ and $R$ are symmetric and positive definite, we know that there exists a unique positive definite solution $X$ such that the system is stabilized with the state feedback law

$$
u(k)=F x(k)
$$

where the feedback gain is given by

$$
F=-\left(R+B^{T} X B\right)^{-1} B^{T} X A
$$

We wish to reduce the dimension of the DARE to be solved and obtain the positive definite solution $X$ in an efficient way. Note that in this situation the structure algorithm reported in [8] cannot be utilized for the sate space reduction.

It has been shown in [7] that state space reduction can be done if the coefficient matrices $A$ and $Q$ of the DARE corresponds to the DLQR problem for a unilateral time-delay system, i.e., a system with time delays along the signal flow.

The unilateral time-delay system considered in [7] consists of $p$ subsystems, where the $i$-th subsystem $(i=$ $1, \ldots, p)$ is described by

$$
\begin{aligned}
x_{i}(k+1) & =A_{i} x_{i}(k)+B_{i} u_{i}(k) \\
y_{i}(k) & =C_{i} x_{i}(k)
\end{aligned}
$$

The subsystems are interconnected with time delay of $d_{i}$ steps, i.e.,

$$
u_{i+1}(k)=y_{i}\left(k-d_{i}\right)
$$

for $i=1, \ldots, p$. The input $u(k)$ to the whole system is applied to the first subsystem via a time delay of $d_{0}$ steps. The output $y(k)$ of the whole system could be defined as

$$
y(k)=u_{p+1}(k)
$$

but it is irrelevant as far as the state feedback is considered.

The whole unilateral time-delay system ca be described in the form of equation (3) with the coefficient matrices:

$$
\begin{gathered}
A=\left[\begin{array}{cccccc}
F_{p} & G_{p} C_{p} & 0 & \cdots & 0 & 0 \\
0 & A_{p} & B_{p} H_{p-1} & \ddots & 0 & 0 \\
\vdots & \ddots & \ddots & \ddots & \ddots & \vdots \\
0 & 0 & \ddots & F_{1} & G_{1} C_{1} & 0 \\
0 & 0 & \cdots & 0 & A_{1} & B_{1} H_{0} \\
0 & 0 & \cdots & 0 & 0 & F_{0}
\end{array}\right] \\
B=\left[\begin{array}{c}
0 \\
0 \\
\vdots \\
0 \\
0 \\
G_{0}
\end{array}\right]
\end{gathered}
$$

Here, $A_{i}, B_{i}, C_{i}(i=1, \ldots, p)$ represent the coefficien$\mathrm{t}$ matrices of the subsystems without time delay, and $F_{i}, G_{i}, H_{i}(i=0, \ldots, p)$ represent those of the timedelay element at the output of $p$-th subsystem, except that $i=0$ corresponds to the time delay at the input of the whole system. More specifically, the coefficient matrices representing the time-delay element have the following form:

$$
\begin{gathered}
F_{i}=\left[\begin{array}{cccc}
0 & I & \cdots & 0 \\
0 & \ddots & \ddots & \vdots \\
\vdots & \ddots & \ddots & I \\
0 & \cdots & 0 & 0
\end{array}\right], \quad G_{i}=\left[\begin{array}{c}
0 \\
\vdots \\
0 \\
I
\end{array}\right] \\
H_{i}=\left[\begin{array}{lllll}
I & 0 & \cdots & \cdots & 0
\end{array}\right]
\end{gathered}
$$

The size of each identity matrix $I$ in $F_{i}, G_{i}, H_{i}$ equals the dimension of the signal connecting the subsystems, and the number of row and column blocks in $F_{i}$ represent the length of the time delay. These numbers may be different for each $i=0, \ldots, p$.

The weighting matrix $Q$ for the unilateral time-delay system is assumed to be given in the following block diagonal form:

$$
Q=\text { block diag }\left(Q_{F_{p}}, Q_{A_{p}}, \ldots, Q_{F_{1}}, Q_{A_{1}}, Q_{F_{0}}\right)
$$

Here, each $Q_{F_{i}}$ for $(i=0, \ldots, p)$ is assumed to be block diagonal, where the size of each block equals the dimension of the signal connecting the subsystems. Each block within $Q_{F_{i}}$ is assumed to be diagonal, thus $Q_{F_{i}}$ turns out to be diagonal as well. 
For this unilateral time-delay system, we consider a fictitious delay-free system described by

$$
\bar{x}(k+1)=\bar{A} \bar{x}(k)+\bar{B} \bar{u}(k), \quad \bar{x}(0)=\bar{x}_{0}
$$

whose coefficient matrices are given by

$$
\bar{A}=\left[\begin{array}{cccc}
A_{p} & B_{p} C_{p-1} & \cdots & 0 \\
0 & \ddots & \ddots & \vdots \\
\vdots & \ddots & A_{2} & B_{2} C_{1} \\
0 & \cdots & 0 & A_{1}
\end{array}\right], \quad \bar{B}=\left[\begin{array}{c}
0 \\
\vdots \\
0 \\
B_{1}
\end{array}\right]
$$

Accordingly, we pose the performance index for the fictitious system as

$$
\bar{J}=\sum_{k=0}^{\infty}\left\{\bar{x}^{T}(k) \bar{Q} \bar{x}(k)+\bar{u}^{T}(k) \bar{R} \bar{u}(k)\right\}
$$

The weighting matrices are determined by

$$
\begin{gathered}
\bar{Q}=\text { block diag }\left(\bar{Q}_{p}, \ldots, \bar{Q}_{1}\right) \\
\bar{Q}_{i}=Q_{A_{i}}+C_{i}^{T} \bar{Q}_{F_{i}} C_{i} \\
\bar{R}=R+\bar{Q}_{F_{0}}
\end{gathered}
$$

where $\bar{Q}_{F_{i}}$ is the sum of the block diagonal elements in $Q_{F_{1}}$.

The DARE to be considered here is

$$
\bar{X}=\bar{A}^{T} \bar{X} \bar{A}+\bar{Q}-\bar{A}^{T} \bar{X} \bar{B}\left(\bar{R}+\bar{B}^{T} \bar{X} \bar{B}\right)^{-1} \bar{B}^{T} \bar{X} \bar{A}
$$

Let us define the following rectangular matrix $T$ which has as many rows as that of $\bar{A}$ and as many columns as that of $A$.

$$
T=\left[\begin{array}{c}
T_{p} A^{\bar{d}_{p}} \\
\vdots \\
T_{2} A^{\bar{d}_{2}} \\
T_{1} A^{\bar{d}_{1}}
\end{array}\right]
$$

Here, $\bar{d}_{i}(i=1, \ldots, p)$ is an integer which equals the total length of time delay up to the $i$-th subsystem, and $T_{i}(i=1, \ldots, p)$ is defined by

$$
T_{i}=\left[\begin{array}{lllllll}
0 & \ldots & 0 & I & 0 & \ldots & 0
\end{array}\right]
$$

The identity matrix $I$ in $T_{i}$ appears at the position corresponding to the $i$-th subsystem in $A$.

We also define a block diagonal matrix $Q_{F}$ as follows:

$$
Q_{F}=\text { block diag }\left(\tilde{Q}_{F_{p}}, 0, \ldots, \tilde{Q}_{F_{1}}, 0, \tilde{Q}_{F_{0}}\right)
$$

Here, the zero block correspond to each subsystem and $\tilde{Q}_{F_{1}}(i=0, \ldots, p)$ correspond to time-delay elements in the whole system. Each $\tilde{Q}_{F_{i}}$ is block diagonal, where the $j$-th block within $\tilde{Q}_{F_{i}}$ is the sum of the blocks within $Q_{F_{i}}$ up to $j$-th position.

With the above definition of the matrices, we have the following formula[7] which connects the solution $X$ to the original DARE (1) and the solution $\bar{X}$ of the smaller DARE (18).

$$
X=T^{T} \bar{X} T+\sum_{i=1}^{p} \sum_{j=0}^{\bar{d}_{i}-1}\left(T_{i} A^{j}\right)^{T} \bar{Q}_{i}\left(T_{i} A^{j}\right)+Q_{F}
$$

This formula relies on the structure of $A$ and $Q$. A single subsystem with time-delay in its input or output is a special case of the unilateral time-delay system, but the singularity of $A$ may arise in a different way. Also, the diagonal weighting on the state variables representing the time-delay element may be restrictive in that the same structure may not be preserved if a similarity transformation is applied to to the state equation. We consider more general case in the following section.

\section{State Space Reduction}

In this section no special structure is assumed for the coefficient matrix $A$. The only assumption is that it is singular. We start by employing an orthogonal transformation such that $A$, and $B$ will take the following form:

$$
A=\left[\begin{array}{ll}
0 & A_{12} \\
0 & A_{22}
\end{array}\right], \quad B=\left[\begin{array}{l}
B_{1} \\
B_{2}
\end{array}\right]
$$

Here the lower right block $A_{22}$ may be singular, depending the Jordan structure of the zero eigenvalues. The weighting matrix $Q$ is transformed and partitioned accordingly, as

$$
Q=\left[\begin{array}{ll}
Q_{11} & Q_{12} \\
Q_{12}^{T} & Q_{22}
\end{array}\right]
$$

The only assumption for $Q$ is that it is symmetric and positive definite. We assume in the following that the coefficient matrices $A, B, Q$ are given in the above form without loss of generality.

Then, we pose a DARE and corresponding DLQR problem whose coefficient matrices are smaller in size, i.e., the size of $A_{22}$ instead of that of $A$. The smaller DARE to be considered is given by

$$
\begin{aligned}
\bar{X}= & \bar{A}^{T} \bar{X} \bar{A}+\bar{Q} \\
& -\left(\bar{S}^{T}+\bar{A}^{T} \bar{X} \bar{B}\right)\left(\bar{R}+\bar{B}^{T} \bar{X} \bar{B}\right)^{-1}\left(\bar{S}+\bar{B}^{T} \bar{X} \bar{A}\right)
\end{aligned}
$$


where the coefficient matrices are defined as follows:

$$
\begin{aligned}
& \bar{A}=A_{22} \\
& \bar{B}=B_{2} \\
& \bar{Q}=A_{12}^{T} Q_{11} A_{12}+A_{12}^{T} Q_{12} A_{22}+A_{22}^{T} Q_{12}^{T} A_{12}+Q_{22} \\
& \bar{S}=A_{12}^{T} Q_{11} B_{1}+A_{12}^{T} Q_{12} B_{2}+A_{22}^{T} Q_{12}^{T} B_{1} \\
& \bar{R}=B_{1}^{T} Q_{11} B_{1}+B_{1}^{T} Q_{12} B_{2}+B_{2}^{T} Q_{12}^{T} B_{1}+R
\end{aligned}
$$

In order to describe the corresponding DLQR problem, we partition the state vector $x(k)$ and its initial value $x_{0}$ in (3) as

$$
x(k)=\left[\begin{array}{l}
x_{1}(k) \\
x_{2}(k)
\end{array}\right], \quad x_{0}=\left[\begin{array}{l}
x_{10} \\
x_{20}
\end{array}\right]
$$

The smaller DLQR problem is to minimize

$$
\bar{J}=\sum_{k=0}^{\infty}\left\{x_{2}^{T}(k) \bar{Q} x_{2}(k)+2 x_{2}^{T}(k) \bar{S} u(k)+u^{T}(k) \bar{R} u(k)\right\}
$$

with respect to the state equation

$$
x_{2}(k+1)=A_{22} x_{2}(k)+B_{2} u(k) ; \quad x_{2}(0)=x_{20}
$$

The smaller DARE has a more general form than the original DARE, due to the existence of the coefficient matrix $\bar{S}$. Accordingly, there is a cross-product ter$\mathrm{m}$ in the performance index $\bar{J}$ with the coefficient $\bar{S}$. Furthermore, the optimal feedback gain $\bar{F}$ is given by

$$
\bar{F}=-\left(\bar{R}+\bar{B}^{T} \bar{X} \bar{B}\right)^{-1}\left(\bar{S}^{T}+\bar{B}^{T} \bar{X} \bar{A}\right)
$$

Now, the comparison of the original and smaller DARE leads to the following relationship.

Lemma 1 The solution $X$ of the original DARE is expressed in terms of the solution $\bar{X}$ of the smaller DARE as

$$
X=\left[\begin{array}{cc}
Q_{11} & Q_{12} \\
Q_{12}^{T} & \bar{X}
\end{array}\right]
$$

Proof First, partition the solution $X$ according to that of (23) as

$$
X=\left[\begin{array}{ll}
X_{11} & X_{12} \\
X_{12}^{T} & X_{22}
\end{array}\right]
$$

Substituting (23) into the (1) and expanding each block immediately shows that $X_{11}=Q_{11}$ and $X_{12}=Q_{12}$. After some manipulation, we have $X_{22}=\bar{X}$.

Thus we only need to solve the smaller DARE (25) in order to obtain a solution $X$ to the original DARE (1).
Note that the smaller DARE has more general form, i.e., it reduces to the same form if $\bar{S}=0$.

Apart from time delays and DARE, a similar reduction method concerning the finite-time DLQR problems has been reported in [9], where the constant directions are identified in order to reduce the state space dimension of the corresponding Riccati difference equation.

Unlike the Riccati difference equation treated in [9], DARE have many solutions in general. The relationship (31) implies that for every solution $X$ of the original DARE, the sub-matrix $\bar{X}$ satisfies the smaller DARE. Conversely, if $\bar{X}$ is a solution to the smaller DARE, the matrix $X$ given by (31) satisfies the original DARE. Thus the original and smaller DARE always have the same number of solutions, despite the difference in the size of the coefficient matrices. We are interested in identifying the solution $\bar{X}$ which corresponds to the unique positive definite solution $X$ of the original DARE.

Although the original and smaller DARE have the same number of solutions, The uniqueness of the positive definite solution for the original DARE does not imply that of the smaller DARE in general. The smaller DARE has a unique positive definite solution $\bar{X}$ if the matrix

$$
\hat{Q}:=\left[\begin{array}{cc}
\bar{Q} & \bar{S} \\
\bar{S}^{T} & \bar{R}
\end{array}\right]
$$

is positive definite, but this does not hold in general.

The following are immediate from Lemma 1 , since $\bar{X}$ in (31) is a principal sub-matrix of $X$.

Remark 1 If the original DARE has a unique positive definite solution $X$, the smaller DARE has at least one positive definite solution $\bar{X}$.

Remark 2 If $Q_{12}=0$ in the original DLQR problem, and the corresponding DARE has a unique positive definite solution $X$, the smaller DARE also has a unique positive definite solution $\bar{X}$. The usually is the case when the DLQR problem for systems with time delay is considered as in [9].

Here, we investigate the performance indices of the original and smaller DLQR problem defined in accordance with the original and smaller DARE, respectively.

We first note that the sub-vector $x_{1}$ does not appear on the right-hand side of the state equation, due to the 
structure of the coefficient $A$. Thus $x_{1}(k)$ for $k>0$ can be eliminated from the expression of $J$. Manipulating $J$ yields

$$
\begin{aligned}
J= & \sum_{k=0}^{\infty}\left\{x_{2}^{T}(k) \bar{Q} x_{2}(k)+2 x_{2}^{T}(k) \bar{S} u(k)+u^{T}(k) \bar{R} u(k)\right\} \\
& +x_{0}^{T}\left[\begin{array}{cc}
Q_{11} & Q_{12} \\
Q_{12}^{T} & 0
\end{array}\right] x_{0}
\end{aligned}
$$

where $\bar{Q}, \bar{S}, \bar{R}$ turn out to be those defined in (26).

Substituting (28) into (34), we have

$$
J=\bar{J}+x_{0}^{T}\left[\begin{array}{cc}
Q_{11} & Q_{12} \\
Q_{12}^{T} & 0
\end{array}\right] x_{0}
$$

where the second term of the right-hand side is determined by the initial value of the original system and not affected by the choice of the control variable $u(k)$.

This leads to the following result.

Theorem 1 If the original DARE has a unique positive definite solution $X$, the smaller DARE has a $u$ nique positive definite solution $\bar{X}$ which corresponding to $X$ and stabilizes the system (29) with the optimal feedback gain $\bar{F}$ given by (30).

Proof It follows from (35) that the existence of the lower bound on $J$ implies that of $\bar{J}$ and that the smaller system (29) is indeed stabilized by the optimal feedback, even if the positive definiteness of $\hat{Q}$ defined in (33) is not guaranteed.

Thus we can utilize existing software which enable us to calculate the stabilizing solution without assuming positive definiteness or non-singularity of the coefficient matrices.

If the matrix $\bar{R}$ is nonsingular, we can define

$$
\begin{aligned}
& \tilde{A}=A_{22}-B_{2} \bar{R}^{-1} \bar{S}^{T} \\
& \tilde{Q}=\bar{Q}-\bar{S} \bar{R}^{-1} \bar{S}^{T}
\end{aligned}
$$

and convert the smaller DARE and corresponding DLQR problem into the same form as the original ones. It may be not be desirable to perform the above conversion if $\bar{R}$ is close to singular. On the other hand, further reduction may be possible if $\bar{R}$ is well conditioned and $\tilde{A}$ turns out to be singular.

Numerical algorithms which accommodate singularity of $R$ generally demand manipulation of larger block matrices. It may be advisable to compare the original DARE and smaller DARE for possible tradeoff between the condition number and size of the coefficient matrices.

\section{Numerical example}

The main result is applied to the following numerical example to illustrate how the size of the Riccati equation to be solved is reduced. This is a simple case where the smaller DARE reduces to the same form as the original DARE.

Let us consider a four-state, two-input discrete-time system with the following coefficient matrices:

$$
\begin{gathered}
A=\left[\begin{array}{cc:cc}
0 & 0 & 1 & 0 \\
0 & 0 & 0 & 1 \\
\hdashline 0 & 0 & 0 & 0 \\
0 & 0 & 0 & 0
\end{array}\right]=:\left[\begin{array}{ll}
0 & A_{12} \\
0 & A_{22}
\end{array}\right] \\
B=\left[\begin{array}{cc}
1 & 0 \\
0 & 1 \\
\hdashline 1 & 0 \\
0 & 1
\end{array}\right]=:\left[\begin{array}{l}
B_{1} \\
B_{2}
\end{array}\right]
\end{gathered}
$$

The pair $(A, B)$ is clearly controllable. We have chosen the matrix $A$ to be in the form of (23) for the sake of simplicity, thus we can partition $A$ and $B$ as indicated by the dashed lines.

Suppose the weighting matrices $Q, R$ of the performance index (2) are given as follows:

$$
\begin{gathered}
Q=\left[\begin{array}{cc:cc}
1 & 0 & 0 & 0 \\
0 & 1 & 0 & 0 \\
\hdashline 0 & 0 & 1 & 0 \\
0 & 0 & 0 & 1
\end{array}\right]=:\left[\begin{array}{ll}
Q_{11} & Q_{12} \\
Q_{12}^{T} & Q_{22}
\end{array}\right] \\
R=\left[\begin{array}{ll}
1 & 0 \\
0 & 1
\end{array}\right]
\end{gathered}
$$

The matrices $Q$ and $R$ are positive definite, thus there exists a unique optimal control for this system. The weighting matrix $Q$ is also partitioned as indicated by the dashed lines. The DARE with above coefficien$t$ matrices may be directly solved using a generalized eigenvector approach, but it may suffer from numerical point of view, due to the existence of repeated poles at the origin.

Substituting the above values into (26) yields

$$
\bar{Q}=\left[\begin{array}{ll}
2 & 0 \\
0 & 2
\end{array}\right], \quad \bar{S}=\left[\begin{array}{ll}
1 & 0 \\
0 & 1
\end{array}\right], \quad \bar{R}=\left[\begin{array}{ll}
2 & 0 \\
0 & 2
\end{array}\right]
$$


and substituting further into (36) yields

$$
\tilde{A}=A_{22}-B_{2} \bar{R}^{-1} \bar{S}^{T}=\left[\begin{array}{cc}
-1 / 2 & 0 \\
0 & -1 / 2
\end{array}\right]
$$

and

$$
\tilde{Q}=\bar{Q}-\bar{S} \bar{R}^{-1} \bar{S}^{T}=\left[\begin{array}{cc}
3 / 2 & 0 \\
0 & 3 / 2
\end{array}\right]
$$

It may be noted that $\tilde{A}$ is nonsingular, whereas $A_{22}$ is a zero matrix.

Now we can solve the Riccati equation (25) in a usual way. In fact we can'solve the Riccati equation without a. computer for this particular case, and the positive definite solution turns out to be

$$
\bar{X}=\left[\begin{array}{cc}
\sqrt{3} & 0 \\
0 & \sqrt{3}
\end{array}\right]
$$

Then the solution of the original DARE is obtained by (31) as:

$$
X=\left[\begin{array}{cc}
Q_{11} & Q_{12} \\
Q_{12}^{T} & \bar{X}
\end{array}\right]=\left[\begin{array}{cc:cc}
1 & 0 & 0 & 0 \\
0 & 1 & 0 & 0 \\
\hdashline 0 & 0 & \sqrt{3} & 0 \\
0 & 0 & 0 & \sqrt{3}
\end{array}\right]
$$

Using the solution $X$, we can calculate the optimal feedback gain

$$
F=\left[\begin{array}{cc:cc}
0 & 0 & -\frac{1}{2+\sqrt{3}} & 0 \\
0 & 0 & 0 & -\frac{1}{2+\sqrt{3}}
\end{array}\right]
$$

and the closed-loop coefficient matrix becomes

$$
A+B F=\left[\begin{array}{cc:cc}
0 & 0 & \frac{1+\sqrt{3}}{2+\sqrt{3}} & 0 \\
0 & 0 & 0 & \frac{1+\sqrt{3}}{2+\sqrt{3}} \\
\hdashline 0 & 0 & \frac{-1}{2+\sqrt{3}} & 0 \\
0 & 0 & 0 & \frac{-1}{2+\sqrt{3}}
\end{array}\right]
$$

The eigenvalues of $A+B F$ are

$$
\{0, \quad 0, \quad-0.2679, \quad-0.2679\}
$$

which verifies that the closed-loop discrete-time system is asymptotically stable. Note that the two poles at the origin have been retained in the close-loop system.

This particular example has been chosen such that the result for unilateral time-delay systems is also applicable. The methods developed for time-delay systems will fail if the off-diagonal blocks of the weighting matrix $Q$ is nonzero, while the method presented here continues to work without problem.

\section{Conclusions}

We studied the discrete algebraic Riccati equation related to the discrete-time linear quadratic regulator problem where the state transition matrix is singular, and derived a method to reduce the size of DARE to be actually solved.

It was clarified that the stabilizing solution of the smaller DARE corresponds to the solution to the original problem, regardless of the positive definiteness or singularities of the coefficient matrices comprising the smaller DARE.

The result is an extension to those developed for unilateral time-delay systems. The present method is directly applicable to discrete-time $\mathcal{H}_{2}$-optimal control problem with singular coefficient $A$ where the stabilizing solution is of interest.

\section{References}

[1] P. Lancaster and L. Rodman: Algebraic Riccati equations, Clarendon Press, Oxford (1995)

[2] S. Bittanti, A. J. Laub, and J. C. Willems (Eds.): The Riccati Equation, Springer-Verlag, Berlin (1991)

[3] V. L. Mehrmann. The autonomous linear quadratic control problem, Lecture notes in control and information sciences 163, Springer-Verlag, Berlin (1991)

[4] V. Ionescu, C. Oară, and M. Weiss: General matrix pencil techniques for the solution of algebraic Riccati equations: a unified approach, IEEE Trans. Autom. Control, AC-42 1085/1097 (1997)

[5] T. Mita: Optimal digital feedback control systems counting computation time of control laws, IEEE Trans. Autom. Control, AC-30 542/548 (1985)

[6] T. Fujinaka and M. Araki: Discrete-time optimal control of systems with unilateral time-delays, $A u$ tomatica, 23 763/765 (1987)

[7] T. Fujinaka and M. Araki: Discrete-time optimal control of unilateral time delay systems (in Japanese), Trans. SICE, 20-4 288/293 (1984)

[8] H. J. Payne and L. M. Silverman: On the discrete time algebraic Riccati equation, IEEE Trans. Autom. Control, AC-18 226/234 (1973)

[9] D. J. Clements and B. D. O. Anderson: Linearquadratic discrete-time control and constant directions, Automatica, 13 255/264 (1977) 